\title{
Vesicle formation in mixture of a PEO-PPO-PEO block copolymer (Pluronic P123) and a nonionic surfactant (Span 65) in water
}

Toshio Sakai, ${ }^{\mathrm{a}, \mathrm{b} *}$ Hiroyoshi Kurosawa, ${ }^{\mathrm{b}}$ Tomohiko Okada ${ }^{\mathrm{b}}$ and Shozi Mishima ${ }^{\mathrm{b}}$

\section{Affiliation:}

${ }^{a}$ International Young Researchers Empowerment Center, Shinshu University, 4-17-1 Wakasato, Nagano 380-8553, Japan

${ }^{\mathrm{b}}$ Department of Chemistry and Material Engineering, Faculty of Engineering, Shinshu University, 4-17-1 Wakasato, Nagano 380-8553, Japan

*Mailing Address:

Department of Chemistry and Material Engineering, Faculty of Engineering, Shinshu University, 4-17-1 Wakasato, Nagano 380-8553, Japan

Phone : +81-26-269-5405

Fax : $+81-26-269-5424$

E-mail : tsakai@shinshu-u.ac.jp 


\begin{abstract}
Fabrication of vesicles with the diameter of 4-30 $\mu \mathrm{m}$ was achieved by mixing of a hydrophobic nonionic surfactant (Span 65) with a poly(ethylene oxide)poly(propylene oxide)-poly(ethylene oxide) block copolymer (Pluronic P123) in aqueous solutions at room temperature. Ring-shaped structure of the Pluronic P123/Span 65 mixture assemblies was confirmed by fluorescence microscopy. The presence of internal aqueous phase in the Pluronic P123/Span 65 mixture assemblies was determined by measuring the entrapment efficiency of calcein using fluorescence spectroscopy. On the other hand, Pluronic P123/Span 60 mixture system formed wire-like assemblies but not vesicles.
\end{abstract}

Key words: Vesicle, Poly(ethylene oxide)-poly(propylene oxide)-poly(ethylene oxide) block copolymer, Nonionic surfactant, Pluronic P123, Span60, Span 65 


\section{Introduction}

Poly(ethylene oxide)-poly(propylene oxide)-poly(ethylene oxide) (PEO-PPOPEO) block copolymers, which are commercial available amphiphilic polymers as Pluronic and Poloxemer, are widely used as dispersion stabilizers [1, 2], pharmaceutical ingredients [3-5], biomedical materials [6,7] and templates for the synthesis of mesoporous materials [8-11] and nanoparticles [12-19]. This is due to a variety of PEO-PPO-PEO block copolymer self-assemblies tuned by molecular characteristics, concentration and temperature in the presence of selective solvents such as water [20, 21]. However, the fabrication of PEO-PPO-PEO block copolymer vesicles is still an important and challenging task because PEO-PPO-PEO block copolymer vesicles formed are not stable in solutions [22-24]. The PEO-PPO-PEO block copolymer vesicles with high stability should provide various opportunities for material, biological and medical applications such as drug delivery system [25]. Thus, several approaches have been attempted to improve the stability of PEO-PPO-PEO block copolymer vesicles, polymer vesicles (polymersomes) and nonionic surfactant vesicles (noisomes): utilization of hydrophobic block copolymers such as poly(ethylene oxide)-poly(ethyl ethylene) [26], poly(ethylene oxide)-cholesterol [27], poly(ethylene oxide)-poly(propylene oxide)-poly(lactic acid) [28] or mixing of polymers, surfactants and hydrophobic components, for example, Pluronic P123/sodium dodcyl sulfate/NaF mixture [29], Pluronic L121/Pluronic P85/pentaerythritol tetraacrylate mixture [30], a poly(ethylene glycol)/Tween 80/Span 80 mixture [31-35], nonionic surfactant/cholesterol mixture [36, 37], poly(ethylene glycol)-b-poly(sodium methacrylate)/cationic surfactant mixture [38], poly(ethylene glycol)-b-poly(acrylic acid)/azobenzene-containing surfactant mixture [39] and poly(ethylene oxide)- $b$-poly(1,2-butylene oxide)/ionic surfactant mixture [40].

In this work, we examined the fabrication of vesicles by mixing of nonionic surfactants with a PEO-PPO-PEO block copolymer in water. We found that mixing of a nonionic surfactant (Span 65) with a PEO-PPO-PEO block copolymer (Pluronic P123) achieved the formation of vesicles with the diameter of 4-30 $\mu \mathrm{m}$ in aqueous solutions. On the other hand, vesicles were not formed in Pluronic P123/Span 60 mixture system but wire-like assemblies were formed.

\section{Experimental}




\subsection{Vesicle preparation}

Conventional chloroform film method [36, 41] was applied for vesicle $\begin{array}{llll}\text { preparation in this } & \text { Pork. Pluronic }\end{array}$ $\left(\mathrm{H}\left[\mathrm{OCH}_{2} \mathrm{CH}_{2}\right]_{19}\left[\mathrm{OCH}_{2} \mathrm{CH}\left(\mathrm{CH}_{3}\right)\right]_{69}\left[\mathrm{OCH}_{2} \mathrm{CH}_{2}\right]_{19} \mathrm{OH} ; \quad \mathrm{EO}_{19} \mathrm{PO}_{68} \mathrm{EO}_{19}, \quad \mathrm{HLB}=8\right.$, BASF), Span 60 (Sorbitan monostearate, $\mathrm{C}_{24} \mathrm{H}_{46} \mathrm{O}_{6}, \mathrm{HLB}=4.7$, Wako), Span 65 (Sorbitan tristearate, $\mathrm{C}_{60} \mathrm{H}_{114} \mathrm{O}_{8}, \mathrm{HLB}=2.1$, Wako) (see Table 1) or their mixture dissolved in chloroform were evaporated at $60{ }^{\circ} \mathrm{C}$ under vacuum by rotary-evaporator. The resulting films composed of the block copolymer, surfactant or block copolymer/surfactant mixture were dried for $24 \mathrm{~h}$ in a desiccator under vacuum at room temperature $\left(\sim 25^{\circ} \mathrm{C}\right)$. The dried film was hydrated and swollen with ultrapure water $\left(18.2 \mathrm{M} \Omega \mathrm{cm}\right.$ at $25{ }^{\circ} \mathrm{C}$, Millipore-filtered water) at $60{ }^{\circ} \mathrm{C}$ for $1 \mathrm{~h}$ following agitation by vortex mixer for $1 \mathrm{~min}$.

\subsection{Characterization}

\subsubsection{Transmittance (turbidity) measurement}

Stability of the assemblies formed was checked by measuring the transmittance (turbidity) of the dispersions with a UV-visible spectrophotometer (U-1900, HITACHI) at a wavelength of $700 \mathrm{~nm}$ in a quartz sample cell with $1 \mathrm{~cm}$ thickness at room temperature $\left(\sim 25^{\circ} \mathrm{C}\right)$. Transmittances of the dispersions were measured at $1 \mathrm{~h}$ after preparation.

\subsubsection{Particle size distribution measurement}

Size distributions of assemblies formed were measured with a laser diffraction particle size distribution analyzer (LA-950, HORIBA). Detectable range is 0.01-3000 $\mu \mathrm{m}$ in diameter.

\subsubsection{Fluorescence microscopy}

Shape of the assemblies formed was confirmed with a fluorescence microscope (BZ9000, KEYENCE). Hydrophobic dye, Coumarin $540\left(\mathrm{C}_{20} \mathrm{H}_{18} \mathrm{~N}_{2} \mathrm{O}_{2} \mathrm{~S}\right.$, Exciton) $\left(\lambda_{\mathrm{ex}}=\right.$ $458 \mathrm{~nm}, \lambda_{\mathrm{em}}=505 \mathrm{~nm}$ ), was solubilized into hydrophobic regions of the assemblies as a fluorescence probe to visualize the assembly structures. The Fluorescence micrographs of the assemblies were observed at 1 day after preparation because it took 1 day to introduce the dye molecules into hydrophobic regions of assemblies.

\subsubsection{Cryo-transmission electron microscopy}


Shape of the assemblies that we obtained was also observed with a cryo-transmission electron microscope (cryo-TEM) (H-7650, Hitachi High Technologies Co.) at an accelerating voltage of $120 \mathrm{kV}$ under a low electron dose at 4 days after preparation. . The specimens for cryo-TEM observation were prepared following a previous report [42]. A small amount of the sample solution was placed on the surface of a TEM copper grid covered by a porous carbon film. The TEM copper grid with the sample solution was held by a pair of self-locking tweezers mounted on a spring-loaded shaft with a cryo preparation system (Leica EM CPC, Leica Microsystems AG). A thin sample film ( $\sim 300 \mathrm{~nm}$ thickness) on the TEM copper grid obtained by blotting the sample solution with a filter paper was plunged into liquid ethane cooled by liquid nitrogen $\left(-175^{\circ} \mathrm{C}\right)$. The TEM copper grid with the frozen sample was transferred onto the tip of a cryospecimen holder (CT3500, Oxford Instruments plc.) under liquid nitrogen. Then, the specimens were observed by the cryo-TEM at $-173{ }^{\circ} \mathrm{C}$.

\subsubsection{Entrapment efficiency measurement}

In order to determine the vesicle formation, the entrapment efficiency of a watersoluble fluorescent maker, calcein $\left(\mathrm{C}_{30} \mathrm{H}_{26} \mathrm{~N}_{2} \mathrm{O}_{13}\right.$, Sigma-Aldrich) $\left(\lambda_{\mathrm{ex}}=490 \mathrm{~nm}, \lambda_{\mathrm{em}}\right.$ $=520 \mathrm{~nm}$ ), in assemblies was measured by a fluorescence method[43] using spectrofluorometer (FP-6200, JASCO). A vesicle contains an internal aqueous phase enclosed by membrane (bilayer) of surfactants and polymers. Thus, formation of vesicles is confirmed by the presence of internal aqueous phase of assemblies which is determined by the entrapment efficiency of calcein in the assemblies [43]. Concentration of the calcein to prepare vesicles was $1 \times 10^{-4} \mathrm{~mol} \mathrm{~L}^{-1}$. The vesicle dispersions were diluted with 500 times by ultrapure water at the measurement of total fluorescence intensity $\left(I_{\text {total }}\right)$. The calcein in the bulk aqueous phase was quenched by complexation with cobalt ions using cobalt chloride $\left(\mathrm{CoCl}_{2}\right.$, SigmaAldrich), and then fluorescence intensity $\left(I_{\text {in }}\right)$ was measured. Subsequently, fluorescence intensity $\left(I_{\mathrm{tx}}\right)$ was measured after the vesicle membrane was ruptured by Triton X-100 $\left(\mathrm{C}_{14} \mathrm{H}_{22} \mathrm{O}\left(\mathrm{C}_{2} \mathrm{H}_{4} \mathrm{O}\right)_{n}, n=9-10\right.$, Sigma-Aldrich). The entrapment efficiency of calcein was calculated according to the following equation:

$$
\text { Entrapment efficiency }(\%)=\frac{I_{\mathrm{in}}-I_{\mathrm{tx}} \times r \times 100}{I_{\mathrm{total}}-I_{\mathrm{tx}} \times r}
$$


where $r$ is the volume correction factor $(r=1.04)$. The fluorescence spectroscopy measurement was performed at $1 \mathrm{~h}$ after preparation.

\section{Results and Discussion}

\subsection{Pluronic P123/Span 60 mixture}

We first checked the stability of Pluronic P123 assemblies formed through hydration and swelling of Pluronic P123 film at $60{ }^{\circ} \mathrm{C}$. An opaque dispersion obtained after hydration and swelling of Pluronic P123 film at $60{ }^{\circ} \mathrm{C}$ changed into a transparent solution by cooling to room temperature $\left(\sim 25^{\circ} \mathrm{C}\right)$ (see Figure 1a). This is due to the transformation of the large assemblies and/or aggregates such as liquid crystal gels formed at $60{ }^{\circ} \mathrm{C}$ into the spherical micelles at room temperature $\left(\sim 25^{\circ} \mathrm{C}\right)$ [44]. Namely, vesicles are not formed from Pluronic P123 alone in aqueous solutions at room temperature $\left(\sim 25^{\circ} \mathrm{C}\right)[22-24]$.

On the other hand, it is known that vesicles are formed in a nonionic surfactant, Span 60, and Span 60/cholesterol mixture (defined as nonionic surfactant vesicles and niosomes) [36]. Opaque dispersions obtained after hydration and swelling of Span 60 and Span 60/cholesterol mixture at $60{ }^{\circ} \mathrm{C}$ remained unchanged even after cooling to room temperature $\left(\sim 25^{\circ} \mathrm{C}\right)$ in the range of 0.1-1.0 Span 60 mass fraction in Span 60/cholesterol mixture (see Figure 1b and Figure 2). A size distribution of the Span 60 assemblies measured by the laser diffraction particle size distribution analyzer at 1 $\mathrm{h}$ after preparation was shown in Figure 3a. Span 60 assemblies with the diameter range of $2-40 \mu \mathrm{m}$ at $1 \mathrm{~h}$ after preparation became smaller with the elapsed time (Figure 3c). The addition of cholesterol into Span 60 led to the size increase of Span60/cholesterol assemblies (see Figure 3b), and sizes of the assemblies hardly changed with the elapsed time for 3 days that we monitored (see Figure 3c). This indicates that Span 60/cholesterol mixture assemblies are stabilized with the aid of cholesterol. These assemblies were observed as bright ring-shaped objects in the diameter range of 2-10 $\mu \mathrm{m}$ by fluorescence microscopy (see Figure 4). The bright ring-shaped objects observed in Figure 4 most likely correspond to the vesicles. Since a vesicle is a spherical hollow capsule composed of membrane (bilayer) of surfactants and polymers, the hydrophobic dye molecules prefer to orient the hydrophobic regions in the membranes of surfactants and polymers. As a result, fluorescence microscope captures the emission from fluorescence dye molecules 
solubilized in the hydrophobic regions of the vesicle membranes. Ring-shaped structure was observed by fluorescence microscope when the diameter of hollowshaped assemblies was larger than the focal depth $(\sim 1 \mu \mathrm{m})$ of the fluorescence microscope. Namely, the hollow-shaped assemblies larger than $\sim 1 \mu \mathrm{m}$ in diameter are observed as the ring-shaped structure by the fluorescence microscopy. Also the objects with smaller diameter than lower detection limit $(\sim 1 \mu \mathrm{m}$ in diameter) cannot be observed by this fluorescence microscope. In addition, vesicle formation of Span 60 and Span 60/cholesterol mixture was confined by measuring the entrapment efficiency of calcein, in which the entrapment efficiency of calcein was estimated to be $\sim 4 \%$ (see Figure 5). This indicates the presence of internal aqueous phase in the Span 60 and Span 60/cholesterol mixture assemblies, that is, formation of vesicles in the Span 60 and Span 60/cholesterol mixture system.

Then, we considered preparing vesicles by mixing of Span 60 with Pluronic P123. Opaque dispersions obtained after hydration and swelling of Pluronic P123/Span 60 mixture at $60{ }^{\circ} \mathrm{C}$ were kept even after cooling to room temperature $\left(\sim 25^{\circ} \mathrm{C}\right)$ in the range of 0.1-1.0 Span 60 mass fraction in Pluronic P123/Span 60 mixture (see Figure 1c and Figure 2). A size distribution with the range of 0.06-0.4 $\mu \mathrm{m}$ of the Pluronic P123/Span 60 mixture assemblies (0.5 Span 60 mass fraction in the mixture) was measured by the laser diffraction particle size distribution analyzer at $1 \mathrm{~h}$ after preparation (see Figure 6a). The addition of Span 60 into Pluronic P123 tends to increase the size of Pluronic P123/Span 60 mixture assemblies (see Figure 6b). The sizes of the assemblies remained unchanged with the elapsed time for 3 days that we monitored (see Figure 6c). No noticeable ring-shaped structure of the Pluronic P123/Span60 mixture assemblies was observed by fluorescence microscopy (see Figure 7). This is most likely due to the smaller sizes of the assemblies than lower detectable limit $(\sim 1 \mu \mathrm{m}$ in diameter) of the fluorescence microscope. In order to confirm the vesicle structure, the entrapment efficiencies of calcein in the Pluronic P123/Span 60 mixture assemblies were measured. Small entrapment efficiencies of calcein in the Pluronic P123/Span 60 mixture assemblies shown in Figure 5 suggest small volume of internal aqueous phase in the Pluronic P123/Span 60 mixture assemblies due to the small-sized assemblies, or no vesicle formation in the Pluronic P123/Span 60 mixture system. Then, cryo-TEM observation was performed for the Pluronic P123/Span 60 mixture assemblies to evaluate the shape of the assemblies 
formed in the Pluronic P123/Span 60 mixture system. We observed wire-like assemblies with the diameter of $\sim 10 \mathrm{~nm}$ and the length of $100-1000 \mathrm{~nm}$ in the cryoTEM images (see Figure 8). Similar-shaped assemblies have been reported by Lof et al.[45] and Schillen et al.[46], in which rod-like micelles have been produced in Pluronic P123/nonionic surfactant $\left(\mathrm{C}_{12} \mathrm{EO}_{6}\right)$ mixture.

Shapes of surfactant- and polymer-assemblies are typically discussed in terms of critical packing parameter (CPP) on the basis of the geometrical structure of surfactant and polymer $[47,48]$. The CPP is given by

$$
\mathrm{CPP}=\frac{v}{a_{0} l_{\mathrm{c}}}
$$

where $v$ is the volume of hydrophobic part (surfactant tail), $a_{0}$ is the area of the hydrophilic part (surfactant head) and $l_{\mathrm{c}}$ is the critical surfactant chain length. It is proposed that spherical micelles, cylindrical micelles, vesicles (flexible bilayers), planar bilayers and inverted micelles are formed at CPP $<1 / 3,1 / 3-1 / 2,1 / 2-1, \sim 1$ and $>1$, respectively [47, 48]. Thus, the formation of Pluronic P123 spherical micelles in an aqueous solution is most likely due to the cone shape of Pluronic P123 critical packing shape $(\mathrm{CPP}<1 / 3)$ (see Table 2). Formation of wire-like assemblies in Pluronic P123/Span 60 mixture system that we observed by cryo-TEM shown in Figure 8 implies that CPP of a Pluronic P123/Span 60 complex would be 1/3-1/2 (see Table 2). That is, Span 60 would not be sufficient hydrophobicity to expand the area of hydrophobic part of the Pluronic P123/Span 60 complex for vesicle formation.

\subsection{Pluronic P123/Span 65 mixture}

We now realized no vesicle formation in the Pluronic P123/Span 60 mixture system. This is most likely due to the small area of the hydrophobic part of the Pluronic P123/Span 60 complex. In order to prepare vesicles in Pluronic P123/nonionic surfactant mixture system, the CPP of a Pluronic P123/nonionic surfactant complex must be 1/2-1 (see Table 2). Namely, more hydrophobic nonionic surfactant should be required to expand the area of hydrophobic part of Pluronic P123/nonionic surfactant complex for vesicle formation of Pluronic P123/nonionic surfactant mixture. 
Then, we next considered mixing Span 65 with Pluronic P123 because Span 65 is a hydrophobic nonionic surfactant more than Span 60 (see Table 1). The Pluronic P123/Span 65 mixture film was hydrated and swollen in water at $60{ }^{\circ} \mathrm{C}$, and the opaque dispersions were retained at room temperature $\left(\sim 25^{\circ} \mathrm{C}\right)$ in the range of 0.1-0.9 Span 65 mass fraction in the Pluronic P123/Span 65 mixture (see Figure 1d and Figure 2). Note here that Span 65 itself does not dissolve in water. A size distribution with the range of 4-30 $\mu \mathrm{m}$ of the Pluronic P123/Span 65 mixture assemblies ( 0.5 Span 60 mass fraction in the mixture) was measured by the laser diffraction particle size distribution analyzer at $1 \mathrm{~h}$ after preparation (see Figure 9a). The size of Pluronic P123/Span65 mixture assemblies was independent on the Span 65 mass fraction in the Pluronic P123/Span 65 mixture assemblies and was kept for 3 days that we monitored after preparation (see Figure 9b). Fluorescence microscopy revealed the ring-shaped structure of Pluronic P123/Span 65 mixture assemblies in the diameter range of 4-10 $\mu \mathrm{m}$ in the range of 0.1-0.9 Span 65 mass fraction in the Pluronic P123/Span 65 mixture (see Figure 10). Since the assemblies were too large to be observed by cryo-TEM, the cryo-TEM image was not shown here. Entrapment efficiencies of calcein in Pluronic P123/Span 65 mixture assemblies were $\sim 5 \%$ (see Figure 5), which is comparable with those of Span 60/cholesterol vesicles. These indicate the formation of vesicles in Pluronic P123/Span 65 mixture system. It should be noted here that cholesterol, which is typically used as a hydrophobic component for fabrication of nonionic surfactant vesicles [36], did not help the formation of Pluronic P123 vesicles.

\section{Conclusions}

We revealed the formation of vesicles by mixing of a hydrophobic nonionic surfactant, Span 65, with a poly(ethylene oxide)-poly(propylene oxide)-poly(ethylene oxide) block copolymer, Pluronic P123. On the other hand, mixture of Span 60 with Pluronic P123 produced the wire-like assemblies but not vesicles. Shape of the assemblies (vesicles and wire-like assemblies) formed by mixing of the nonionic surfactants with Pluronic P123 could be explained in terms of the critical packing parameter (CPP) (geometrical structure) of Pluronic P123/nonionic surfactant complex. Span 65 would expand the hydrophobic part of Pluronic P123/nonionic surfactant complex more than Span 60 because the Span 65 is more hydrophobic than 
Span 60. These findings we reported in this paper should provide better insight on the vesicle formation of poly(ethylene oxide)-poly(propylene oxide) block copolymers and further opportunities in colloidal and biomedical applications (e.g., drug-delivery system and bio-imaging).

\section{Acknowledgements}

This study was performed through Program for Dissemination of Tenure Tracking System funded by the Ministry of Education and Science, Japan. We are grateful to Prof. Koji Tsuchiya at Tokyo University of Science for cryo-TEM observation. 


\section{References}

[1] T.J. Barnes, C.A. Prestidge, PEO-PPO-PEO block copolymers at the emulsion droplet-water interface, Langmuir 16 (2000) 4116-4121.

[2] Y.N. Lin, P. Alexandridis, Temperature-dependent adsorption of pluronic F127 block copolymers onto carbon black particles dispersed in aqueous media, J. Phys. Chem. B 106 (2002) 10834-10844.

[3] L. Yang, P. Alexandridis, Physicochemical aspects of drug delivery and release from polymer-based colloids, Curr. Opin. Colloid Interface Sci. 5 (2000) 132143.

[4] R. Ivanova, B. Lindman, P. Alexandridis, Effect of pharmaceutically acceptable glycols on the stability of the liquid crystalline gels formed by poloxamer 407 in water, J. Colloid Interface Sci. 252 (2002) 226-235.

[5] A.V. Kabanov, P. Lemieux, S. Vinogradov, V. Alakhov, Pluronic((R)) block copolymers: novel functional molecules for gene therapy, Advanced Drug Delivery Reviews 54 (2002) 223-233.

[6] F. Ahmed, P. Alexandridis, H. Shankaran, S. Neelamegham, The ability of poloxamers to inhibit platelet aggregation depends on their physicochemical properties, Thromb. Haemostasis 86 (2001) 1532-1539.

[7] D. Cohn, A. Sosnik, A. Levy, Improved reverse thermo-responsive polymeric systems, Biomaterials 24 (2003) 3707-3714.

[8] D. Zhao, J. Feng, Q. Huo, N. Melosh, G.H. Fredrickson, B.F. Chmelka, G.D. Stucky, Triblock copolymer syntheses of mesoporous silica with periodic 50 to 300 angstrom pores, Science 279 (1998) 548-552.

[9] J.Y. Ying, C.P. Mehnert, M.S. Wong, Synthesis and applications of supramolecular-templated mesoporous materials, Angew. Chem. Int. Ed. 38 (1999) 56-77.

[10] G.J.d.A.A. Soler-Illia, C. Sanchez, B. Lebeau, J. Patarin, Chemical strategies to design textured materials: from microporous and mesoporous oxides to nanonetworks and hierarchical structures, Chem. Rev. 102 (2002) 4093-4138.

[11] G.J.d.A.A. Soler-Illia, E.L. Crepaldi, D. Grosso, C. Sanchez, Block copolymer-templated mesoporous oxides, Curr. Opin. Colloid Interface Sci. 8 (2003) 109-126. 
[12] G.N. Karanikolos, P. Alexandridis, G. Itskos, A. Petrou, T.J. Mountziaris, Synthesis and size control of luminescent ZnSe nanocrystals by a microemulsion-gas contacting technique, Langmuir 20 (2004) 550-553.

[13] J.-U. Kim, S.-H. Cha, K. Shin, J.Y. Jho, J.-C. Lee, Preparation of gold nanowires and nanosheets in bulk block copolymer phases under mild conditions, Adv. Mater. 16 (2004) 459-464.

[14] T. Sakai, P. Alexandridis, Single-step synthesis and stabilization of metal nanoparticles in aqueous Pluronic block copolymer solutions at ambient temperature, Langmuir 20 (2004) 8426-8430.

[15] T. Sakai, P. Alexandridis, Mechanism of gold metal ion reduction, nanoparticle growth and size control in aqueous amphiphilic block copolymer solutions at ambient conditions, J. Phys. Chem. B 109 (2005) 7766-7777.

[16] T. Sakai, P. Alexandridis, Spontaneous formation of gold nanoparticles in poly(ethylene oxide)-poly(propylene oxide) solutions: Solvent quality and polymer structure effects, Langmuir 21 (2005) 8019-8025.

[17] T. Sakai, P. Alexandridis, Ag and au monometallic and bimetallic colloids: Morphogenesis in amphiphilic block copolymer solutions, Chem. Mater. 18 (2006) 2577-2583.

[18] W.K. Lee, S.H. Cha, K.H. Kim, B.W. Kim, J.C. Lee, Shape-controlled synthesis of gold icosahedra and nanoplates using Pluronic P123 block copolymer and sodium chloride, J. Solid State Chem. 182 (2009) 3243-3248.

[19] P. Khullar, A. Mahal, V. Singh, T.S. Banipal, G. Kaur, M.S. Bakshi, How PEO-PPO-PEO triblock polymer micelles control the synthesis of gold nanoparticles: temperature and hydrophobic effects, Langmuir 26 (2010) 11363-11371.

[20] P. Alexandridis, U. Olsson, B. Lindman, A record nine different phases (four cubic, two hexagonal, and one lamellar lyotropic liquid crystalline and two micellar solutions) in a ternary isothermal system of an amphiphilic block copolymer and selective solvents (water and oil), Langmuir 14 (1998) 2627-2638.

[21] R. Ivanova, B. Lindman, P. Alexandridis, Evolution in structural polymorphism of Pluronic F127 poly(ethylene oxide)-poly(propylene oxide) block copolymer in ternary systems with water and pharmaceutically acceptable organic solvents: From "glycols" to "oils", Langmuir 16 (2000) 9058-9069. 
[22] K. Schillen, K. Bryskhe, Y.S. Mel'nikova, Vesicles formed from a poly(ethylene oxide)-poly(propylene oxide)-poly(ethylene oxide) triblock copolymer in dilute aqueous solution, Macromolecules 32 (1999) 6885-6888.

[23] K. Bryskhe, J. Jansson, D. Topgaard, K. Schillen, U. Olsson, Spontaneous vesicle formation in a block copolymer system, J. Phys. Chem. B 108 (2004) 97109719.

[24] J. Zipfel, J. Berghausen, G. Schmidt, P. Lindner, P. Alexandridis, W. Richtering, Influence of shear on solvated amphiphilic block copolymers with lamellar morphology, Macromolecules 35 (2002) 4064-4074.

[25] C.J. Drummond, C. Fong, Surfactant self-assembly objects as novel drug delivery vehicles, Curr. Opin. Colloid Interface Sci. 4 (2000) 449-456.

[26] B.M. Discher, Y.-Y. Won, D.S. Ege, J.C.-M. Lee, F.S. Bates, D.E. Discher, D.A. Hammer, Polymersomes: Tough vesicles made from diblock copolymers, Science 284 (1999) 1143-1146.

[27] Y. Dan-do, Z. Jia-bi, H. Zhang-jian, R. Hai-xia, Z. Zeng-juan, Synthesis and application of poly(ethylene oxide)-cholesterol (Chol-PEGm) conjugates in physicochemical characterization of nonionic surfactant vesicles, Colloids Surf. B 63 (2008) 192-199.

[28] X.Y. Xiong, K.C. Tam, L.H. Gan, Synthesis and aggregation behavior of Pluronic F127/poly(lactic acid) block copolymers in aqueous solutions, Macromolecules 36 (2003) 9979-9985.

[29] S. Chen, B. Yang, C. Guo, J.H. Ma, L.R. Yang, X.F. Liang, C. Hua, H.Z. Liu, Spontaneous vesicle formation of poly(ethylene oxide)-poly(propylene oxide)poly(ethylene oxide) triblock copolymer, J. Phys. Chem. B 112 (2008) 15659-15665.

[30] F. Li, L.H.J. de Haan, A.T.M. Marcelis, F.A.M. Leermakers, M.A.C. Stuart, E.J.R. Sudholter, Pluronic polymersomes stabilized by core cross-linked polymer micelles, Soft Matter 5 (2009) 4042-4046.

[31] T.Q. Liu, R. Guo, Preparation of a highly stable niosome and its hydrotropesolubilization action to drugs, Langmuir 21 (2005) 11034-11039.

[32] T.Q. Liu, R. Guo, Investigation of PEG 6000/Tween 80/Span 80/ $\mathrm{H}_{2} \mathrm{O}$ niosome microstructure, Colloid Polymer Sci. 285 (2007) 711-713.

[33] T.Q. Liu, R. Guo, Structure and transformation of the niosome prepared from PEG 6000/Tween 80/Span 80/ $\mathrm{H}_{2} \mathrm{O}$ lamellar liquid crystal, Colloids Surf. A 295 (2007) 130-134. 
[34] T.Q. Liu, R. Guo, W. Hua, J. Qiu, Structure behaviors of hemoglobin in PEG 6000/Tween 80/Span 80/ $\mathrm{H}_{2} \mathrm{O}$ niosome system, Colloids Surf. A 293 (2007) 255-261.

[35] W. Hua, T.Q. Liu, Preparation and properties of highly stable innocuous niosome in Span 80/PEG 400/ $\mathrm{H}_{2} \mathrm{O}$ system, Colloids Surf. A 302 (2007) 377-382.

[36] A. Manosroi, P. Wongtrakul, J. Manosroi, H. Sakai, F. Sugawara, M. Yuasa, M. Abe, Characterization of vesicles prepared with various non-ionic surfactants mixed with cholesterol, Colloids Surf. B 30 (2003) 129-138.

[37] M. Tabbakhian, N. Tavakoli, M.R. Jaafari, S. Daneshamouz, Enhancement. of follicular delivery of finasteride by liposomes and niosomes - 1. In vitro permeation and in vivo deposition studies using hamster flank and ear models, Inter. J. Pharm. 323 (2006) 1-10.

[38] A.V. Kabanov, T.K. Bronich, V.A. Kabanov, K. Yu, A. Eisenberg, Spontaneous formation of vesicles from complexes of block ionomers and surfactants, J. Am. Chem. Soc. 120 (1998) 9941-9942.

[39] Y.P. Wang, P. Han, H.P. Xu, Z.Q. Wang, X. Zhang, A.V. Kabanov, Photocontrolled self-assembly and disassembly of block ionomer complex vesicles: A facile approach toward supramolecular polymer nanocontainers, Langmuir 26 (2010) 709-715.

[40] A. Kelarakis, V. Castelletto, M.J. Krysmann, V. Havredaki, K. Viras, I.W. Hamley, Polymer-surfactant vesicular complexes in aqueous medium, Langmuir 24 (2008) 3767-3772.

[41] A. Yoshida, A. Manosroi, J. Manosroi, H. Yamauchi, M. Abe, Molecular interactions between phospholipids and mangostin in a lipid bilayer, Colloids Surf. B 4 (1995) 423-432.

[42] T. Sakai, T. Mukawa, K. Tsuchiya, H. Sakai, M. Abe, Facile preparation of gold nanoparticles-liposome composites, J. Nanosci. Nanotechnol. 9 (2009) 461-466.

[43] N. Oku, D.A. Kendall, R.C. MacDonald, A simple procedure for the determination of the trapped volume of liposomes, Biochimica et Biophysica Acta (BBA) - Biomembranes 691 (1982) 332-340.

[44] M. Svensson, P. Alexandridis, P. Linse, Phase behavior and microstructure in binary block copolymer/selective solvent systems: Experiments and theory, Macromolecules 32 (1999) 637-645.

[45] D. Lof, A. Niemiec, K. Schillen, W. Loh, G. Olofsson, A calorimetry and light scattering study of the formation and shape transition of mixed micelles of 
$\mathrm{EO}_{20} \mathrm{PO}_{68} \mathrm{EO}_{20}$ triblock copolymer (P123) and nonionic surfactant $\left(\mathrm{C}_{12} \mathrm{EO}_{6}\right)$, J. Phys. Chem. B 111 (2007) 5911-5920.

[46] K. Schillen, J. Jansson, D. Lof, T. Costa, Mixed micelles of a PEO-PPO-PEO triblock copolymer (P123) and a nonionic surfactant $\left(\mathrm{C}_{12} \mathrm{EO}_{6}\right)$ in water. A dynamic and static light scattering study, J. Phys. Chem. B 112 (2008) 5551-5562.

[47] J.N. Israelachvili, D.J. Mitchell, B.W. Ninham, Theory of self-assembly of hydrocarbon amphiphiles into micelles and bilayers, J. Chem. Soc. Faraday Trans. I 72 (1976).

[48] J. Israelachvili, Intermolecular \& Surface Forces, Academic Press, California, 1985. 
COLSUA-D-11-00484 Revised July 2011

Table 1. Nonionic surfactants that we used in this work

\begin{tabular}{|c|c|c|}
\hline $\begin{array}{c}\text { Nonionic surfactant } \\
\text { Sorbitan monostearate }\end{array}$ & HLB \\
\hline $\begin{array}{c}\text { Sorbitan tristearate } \\
\text { (Span 65) }\end{array}$ & 4.7 \\
\hline
\end{tabular}


Table 2 Schematic illustration of Pluronic P123 and Pluronic P123/nonionic surfactant assemblies

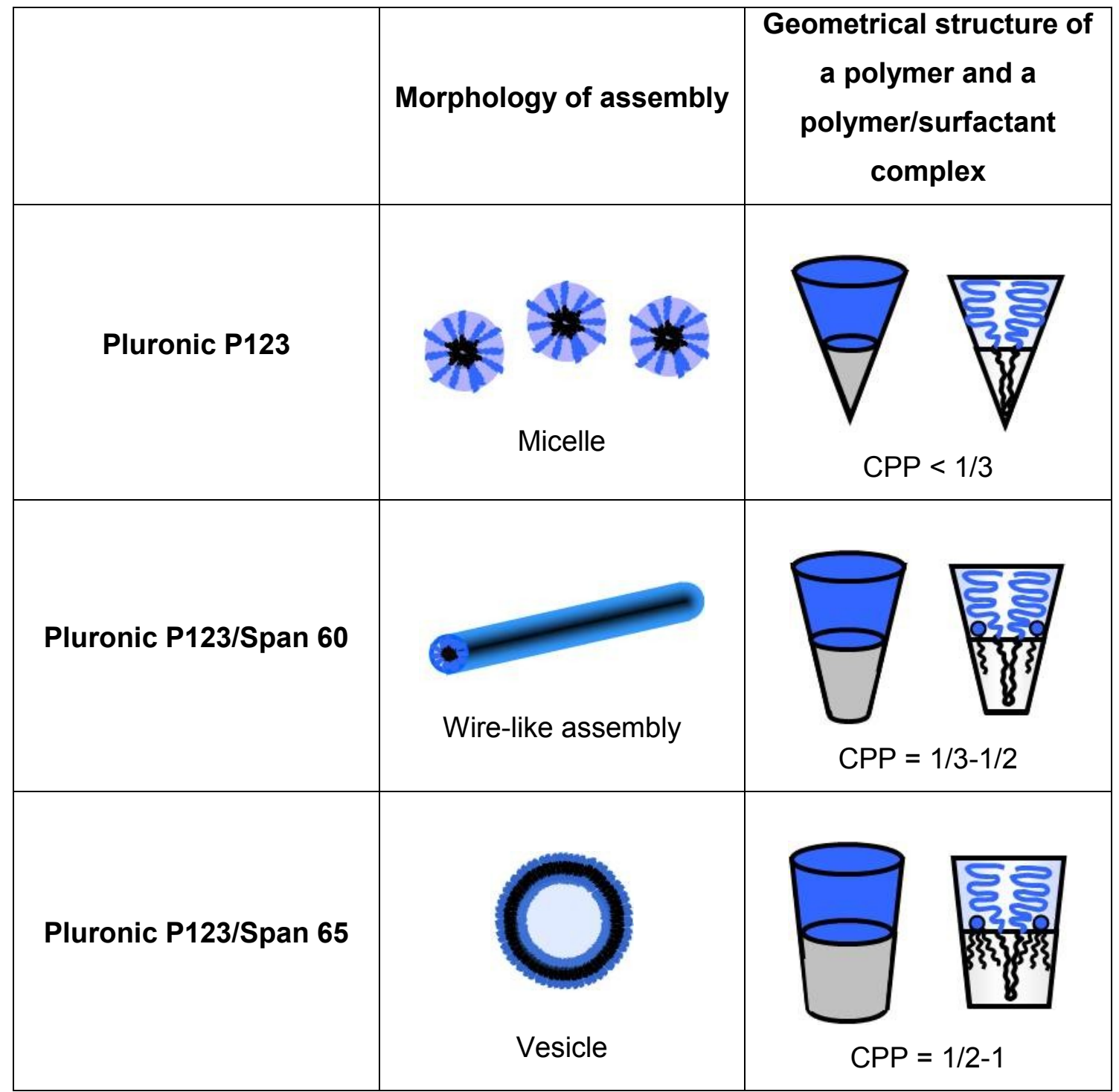




\section{Figure captions}

Figure 1. (a) An opaque dispersion obtained through hydration and swelling of Pluronic P123 film (5 mM) at $60{ }^{\circ} \mathrm{C}$ and a transparent solution of Pluronic P123 obtained after cooling of the opaque dispersion at $60{ }^{\circ} \mathrm{C}$ to room temperature $\left(\sim 25^{\circ} \mathrm{C}\right)$. (b) Dispersions of Span 60 and Span 60/cholesterol mixture, (c) Span 60 and Pluronic P123/Span 60 mixture and (d) Pluronic P123/Span 65 mixture in water at room temperature $\left(\sim 25^{\circ} \mathrm{C}\right)$ observed at $1 \mathrm{~h}$ after preparation.

Figure 2. Transmittances of (०) Span 60/cholesterol mixture, (•) Pluronic P123/Span 60 mixture and ( $\Delta$ ) Pluronic P123/Span 65 mixture dispersions at room temperature $\left(\sim 25{ }^{\circ} \mathrm{C}\right)$ after cooling the dispersions containing the assemblies hydrated and swollen in water at $60{ }^{\circ} \mathrm{C}$, plotted as a function of surfactant (Span 60 or Span 65) mass fraction in the mixtures.

Figure 3. Particle size distributions of (a) Span 60 assemblies and (b) Span 60/cholesterol (cholesterol mass fraction of 0.5 ) mixture assemblies measured at $1 \mathrm{~h}$ after preparation. (c) Average diameters of Span 60 assemblies and Span 60/cholesterol mixture assemblies plotted as a function of the elapsed time. Span 60

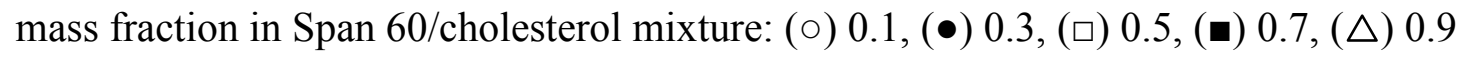
and ( $\mathbf{\Delta})$ 1.0.

Figure 4. Fluorescence micrographs of (a) Span 60 assemblies and (b) Span $60 /$ cholesterol (cholesterol mass fraction of 0.5 ) mixture assemblies formed at room temperature $\left(\sim 25^{\circ} \mathrm{C}\right)$.

Figure 5. Entrapment efficiencies of calcein in (०) Span 60/cholesterol assemblies, (•) Pluronic P123/Span 60 mixture assemblies and (A) Pluronic P123/Span 65 mixture assemblies plotted as a function of surfactant (Span 60 or Span 65) mass fraction in the mixtures at room temperature $\left(\sim 25^{\circ} \mathrm{C}\right)$.

Figure 6. (a) Particle size distribution of the Pluronic P123/Span 60 (Span 60 mass fraction of 0.5 ) mixture assemblies measured at $1 \mathrm{~h}$ after preparation. (b) Average diameters of Span 60 assemblies and Pluronic P123/Span 60 mixture assemblies 
plotted as a function of the elapsed time. Span 60 mass fraction in Pluronic

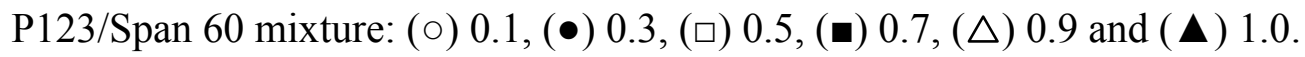

Figure 7. Fluorescence micrograph of Pluronic P123/Span 60 (Span 60 mass fraction of 0.5$)$ mixture assemblies formed at room temperature $\left(\sim 25^{\circ} \mathrm{C}\right)$.

Figure 8. Cryo-TEM images of assemblies formed in Pluronic P123/Span 60 mixture system at room temperature $\left(\sim 25^{\circ} \mathrm{C}\right)$. Span 60 mass fractions of (a) 0.5 and (b) 0.1 in the Pluronic P123/Span 60 mixture. Wire-like assemblies are pointed by black arrows.

Figure 9. (a) Particle size distribution of the Pluronic P123/Span 65 (Span 65 mass fraction of 0.5 ) mixture assemblies measured at $1 \mathrm{~h}$ after preparation. (b) Average diameters of Pluronic P123/Span 65 mixture assemblies plotted as a function of the elapsed time. Span 65 mass fraction in Pluronic P123/Span 65 mixture: (०) 0.1, (•) $0.3,(\square) 0.5,(\mathbf{a}) 0.7$ and $(\triangle) 0.9$.

Figure 10. Fluorescence micrograph of Pluronic P123/Span 65 (Span 65 mass fraction of 0.5$)$ mixture assemblies formed at room temperature $\left(\sim 25^{\circ} \mathrm{C}\right)$. 
Figure 1. Sakai et al.

(a) Pluronic P123 (5 mM)

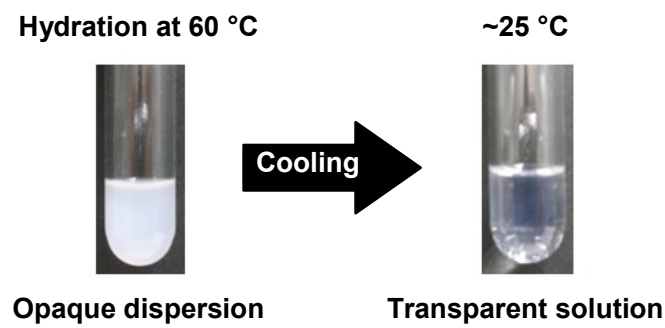

(b)

Span 60 mass fraction in

Span 60/Cholesterol mixture

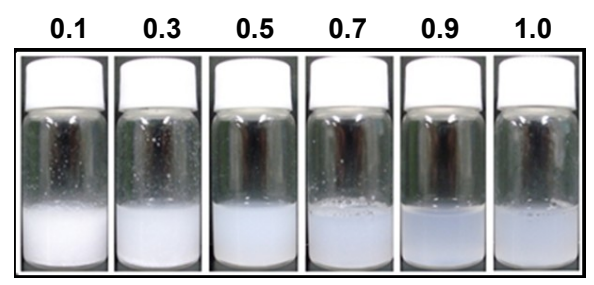

(c)

Span 60 mass fraction in

Pluronic P123/Span 60 mixture

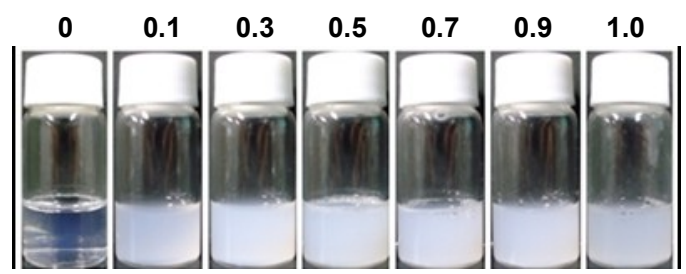

(d)

Span 65 mass fraction in Pluronic P123/Span 65 mixture

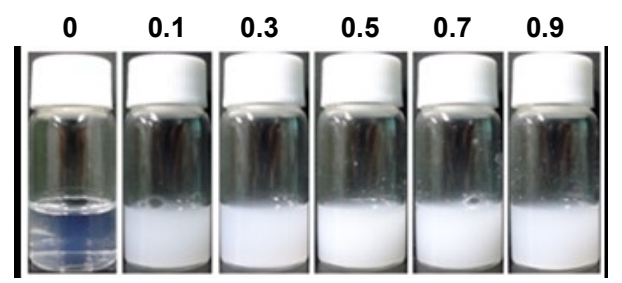


COLSUA-D-11-00484 Revised July 2011

Figure 2. Sakai et al.

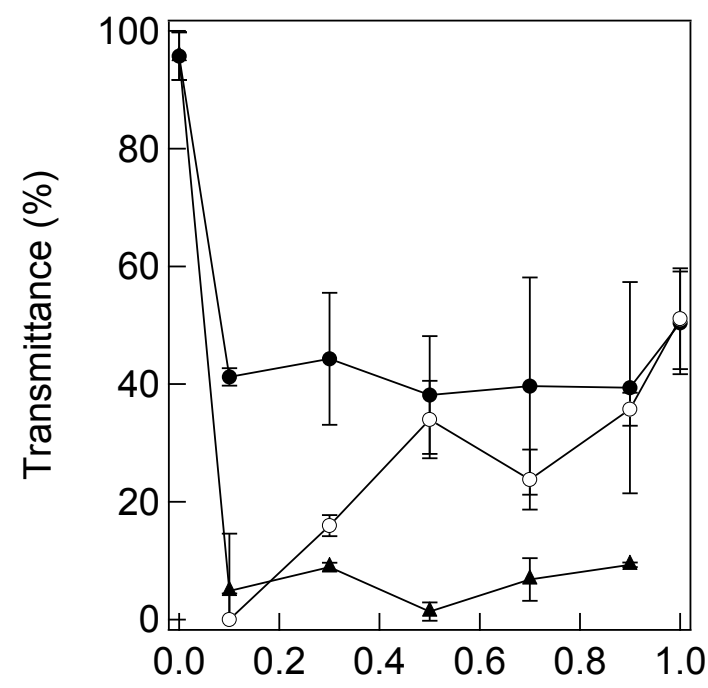

Surfactant concentration (mass fraction) 
Figure 3. Sakai et al.
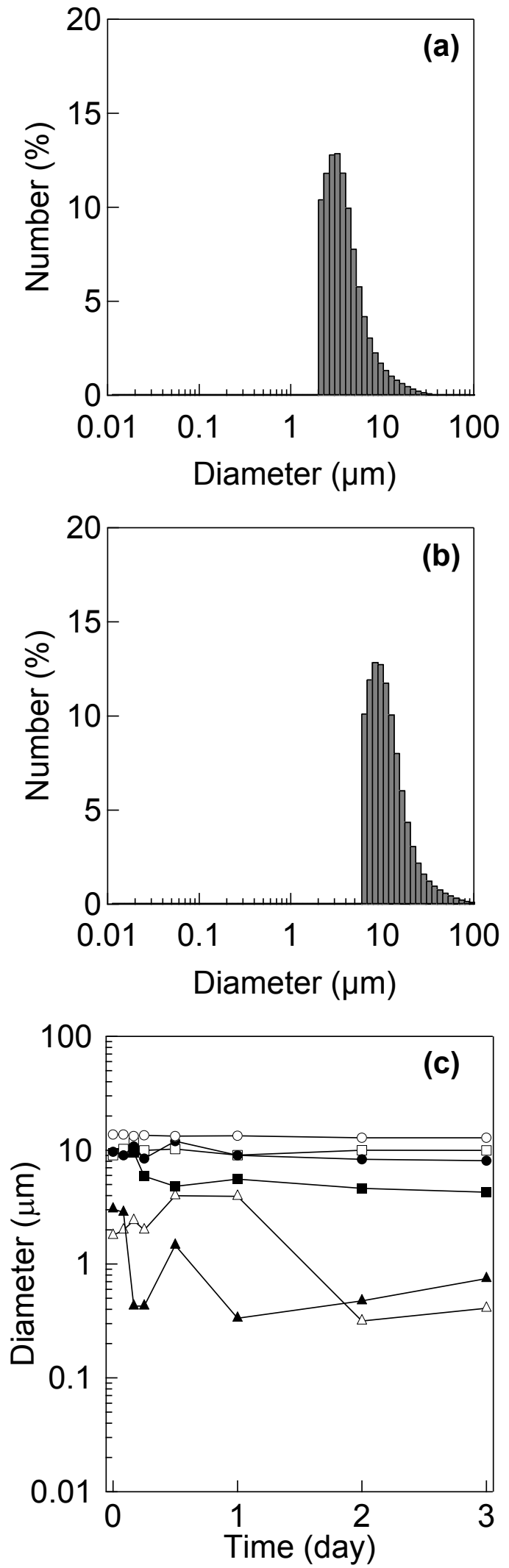
COLSUA-D-11-00484 Revised July 2011

Figure 4. Sakai et al.

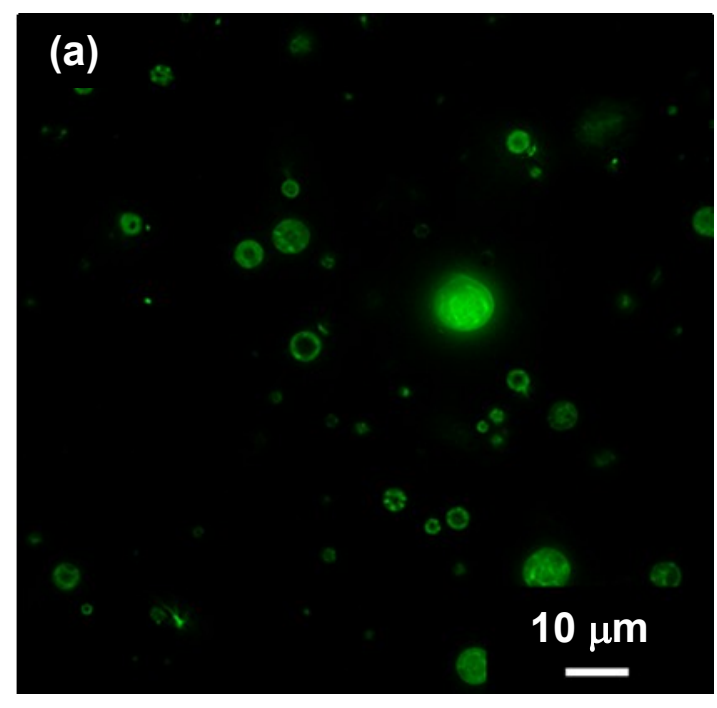

(b)

$10 \mu \mathrm{m}$ 
COLSUA-D-11-00484 Revised July 2011

Figure 5. Sakai et al.

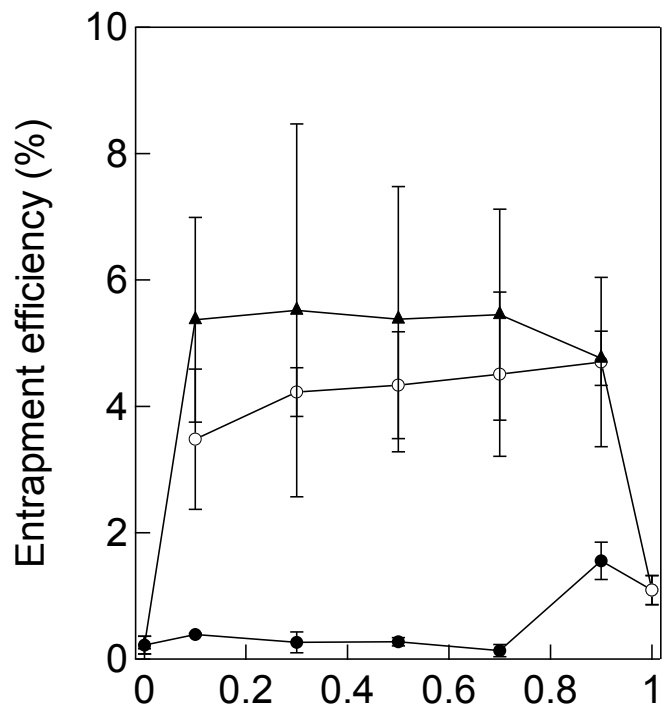

Surfactant concentration (mass fraction) 
COLSUA-D-11-00484 Revised July 2011

Figure 6. Sakai et al.
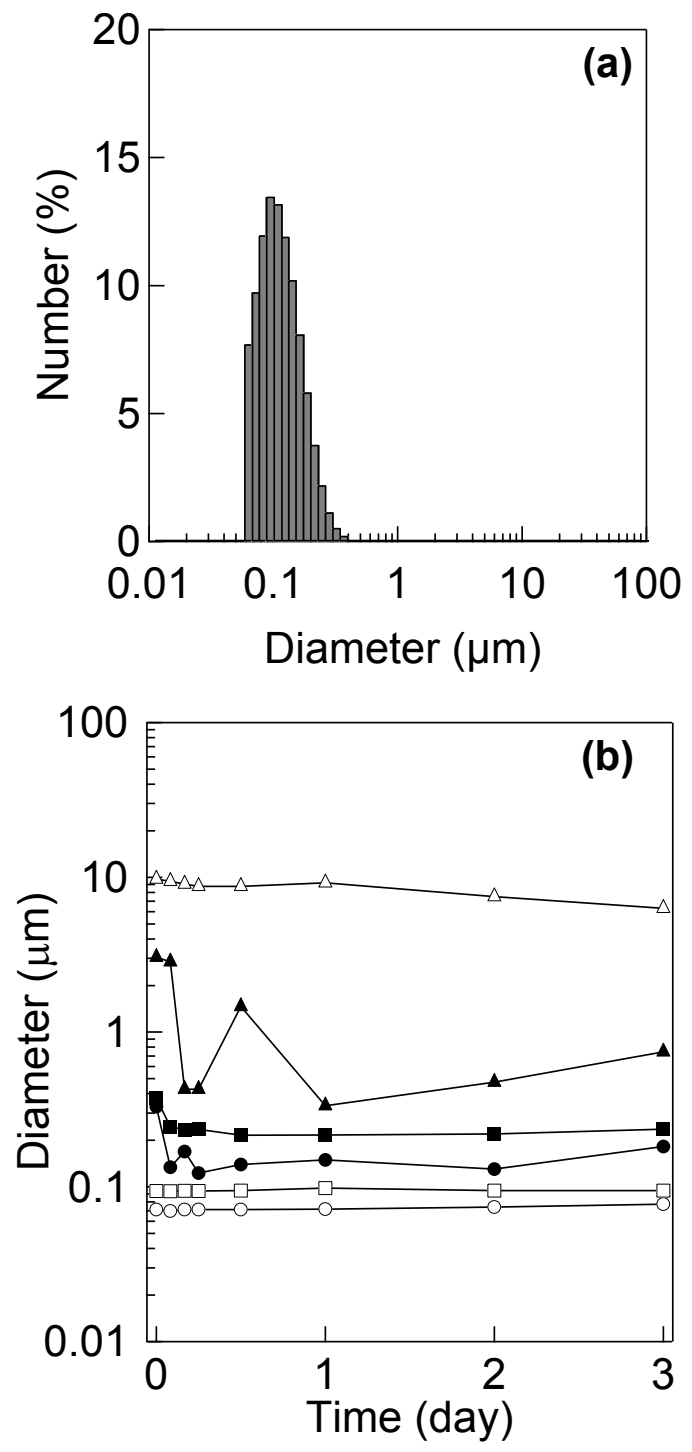
COLSUA-D-11-00484 Revised July 2011

Figure 7. Sakai et al.

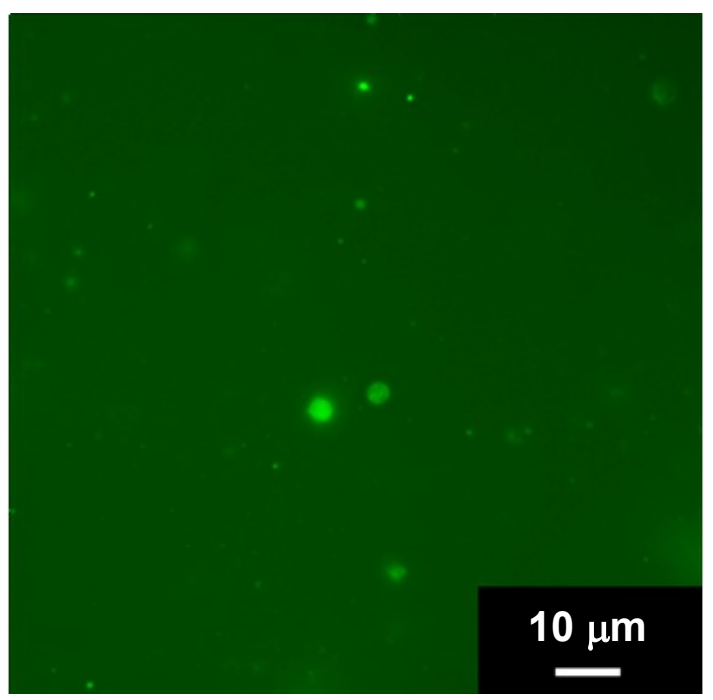


COLSUA-D-11-00484 Revised July 2011

Figure 8. Sakai et al.
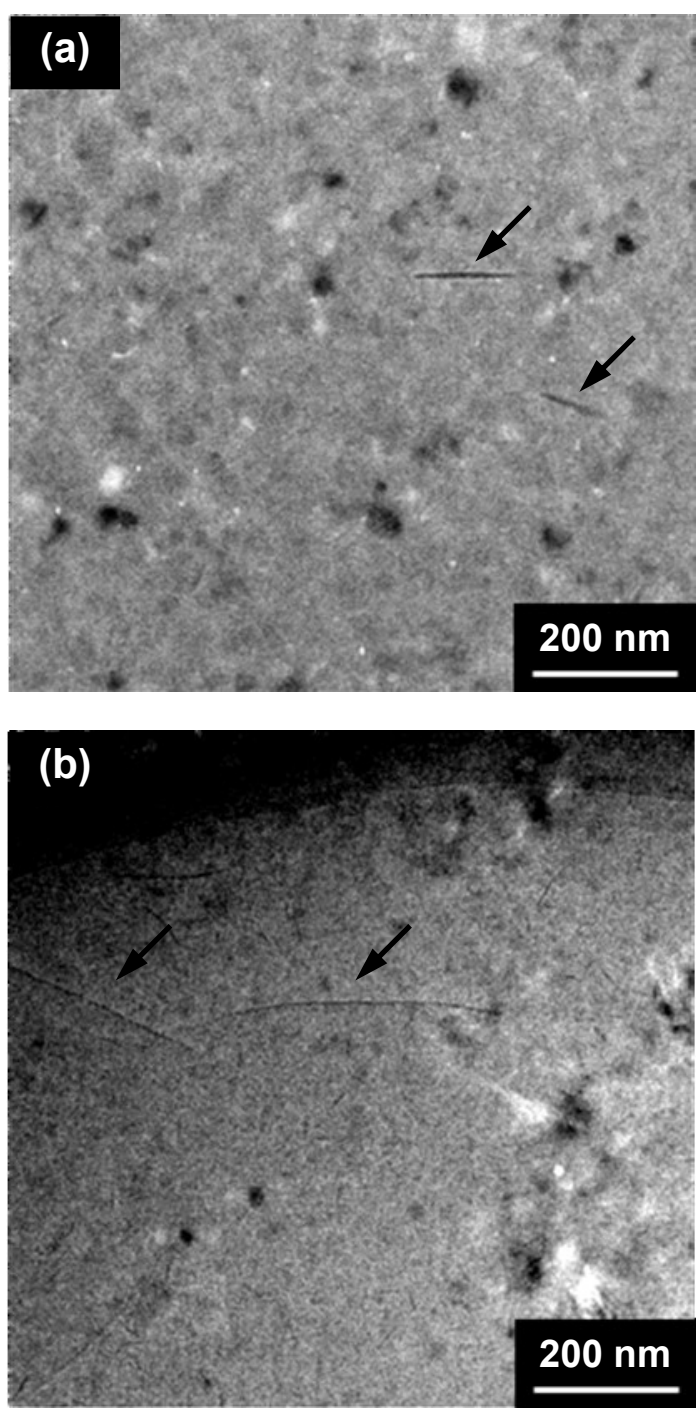
COLSUA-D-11-00484 Revised July 2011

Figure 9. Sakai et al.
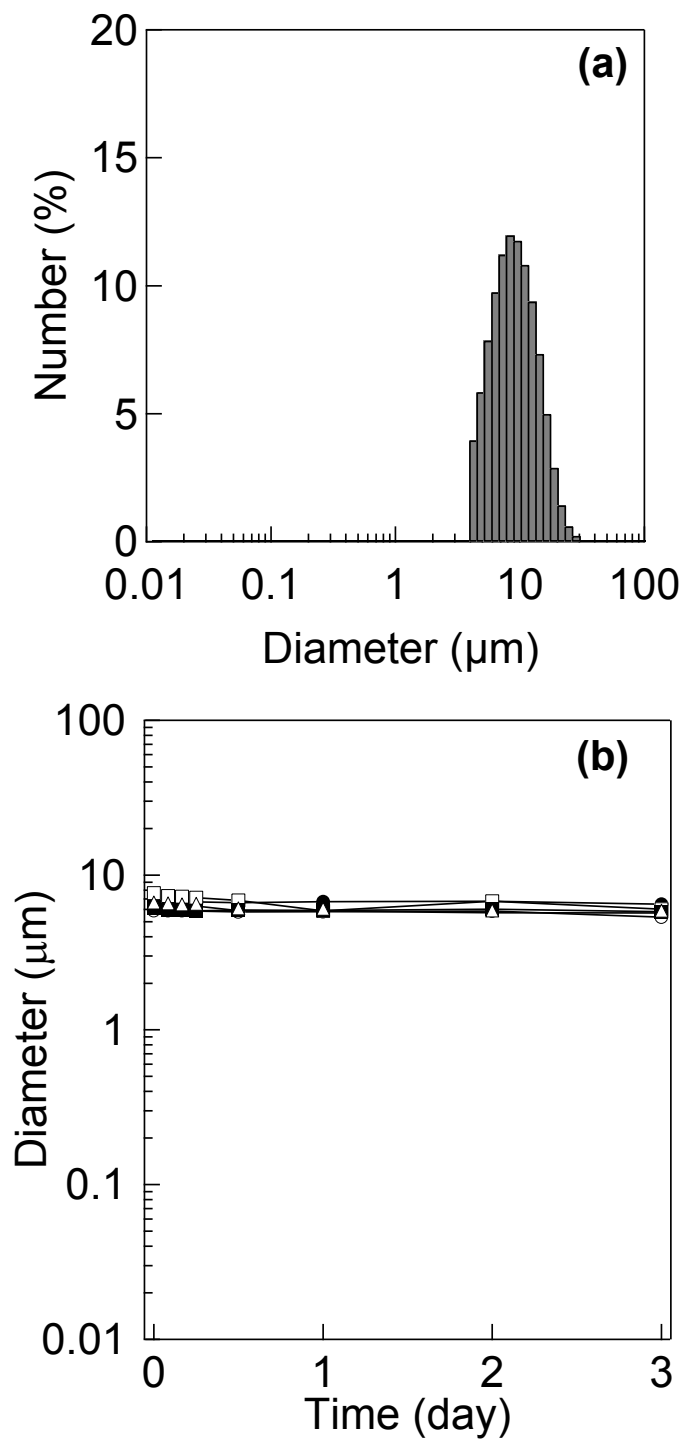
COLSUA-D-11-00484 Revised July 2011

Figure 10. Sakai et al.

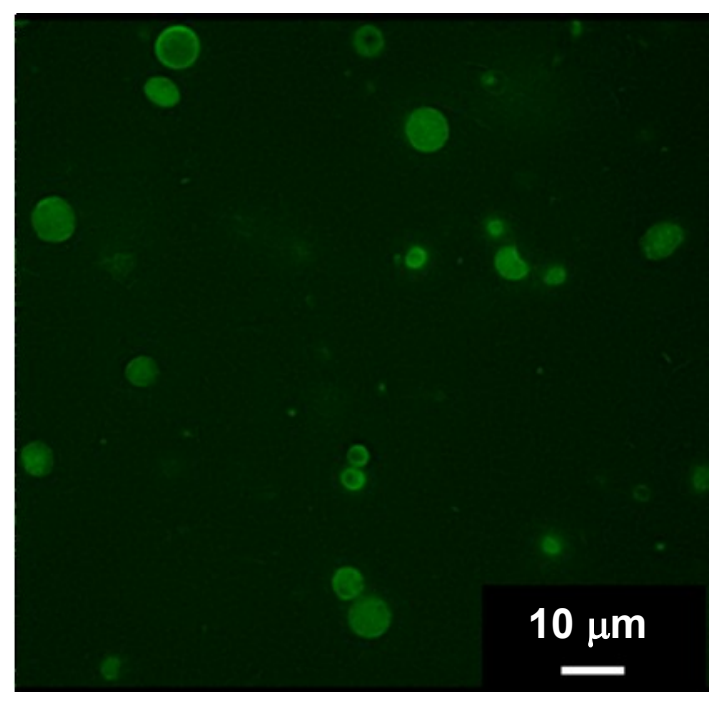

